\title{
Encryption of DES Algorithm in Information Security
}

\author{
${ }^{1}$ Isnar Sumartono, ${ }^{2}$ Andysah Putera Utama Siahaan \\ Faculty of Science and Technology, Universitas Pembangunan Panca Budi, Medan, Indonesia \\ Email: ${ }^{1}$ isnar@pancabudi.ac.id, ${ }^{2}$ andiesiahaan@gmail.com
}

\begin{abstract}
Information security is the protection of personal and non-personal data from various threats to guarantee privacy. For business practices, data security can reduce business risk, and increase the return of investment and business opportunities. In designing information system security systems, there are information security aspects that need to be considered. Many threats will come before the information circulating. Information is a matter that will be targeted by wild parties. Cryptographic algorithms are needed to protect data from these threats. Data Encryption Standard (DES) belongs to the symmetry cryptography system and is classified as a block cipher type. DES operates on 64-bit block size. DES encrypts 64 plaintext bits into 64-bit ciphertext using 56 private key bits or subkeys. The internal key is generated from an external key that is 64 bits long. The DES method is an excellent cryptographic technique used to secure data. DES has 16 rounds to ensure safer data against unexpected attacks. Applying DES to data encryption will be very useful for protecting data.
\end{abstract}

Keywords: DES, encryption, decryption, algorithm.

\section{INTRODUCTION:}

The digital age is an era where data is exchanged through bits on the internet network [1]-[3]. It causes security of the data is not guaranteed. Data can be intercepted during the shipping process. Networks that have been connected to a global network are networks that can be dismantled by anyone [4]. Information is one of the essential assets to be protected by its security. Users need to pay attention to the security of the information assets. Leakage of information and failure of the system can result in losses for users of that information. Data leakage can occur due to intentional or accidental [5]. It will be detrimental to both the financial side and one's productivity [6]. Data security is quality or state of being secure-to be free from danger. Some things that need to be considered to determine the security strategy are as follows:

- Physical Security, a strategy that focuses on securing organizational members, physical assets, unauthorized access and workplaces from various threats including fire hazards

- Personal Security, a more focused strategy to protect people in the organization

- Operation Security, a strategy to secure the ability of an organization or company to work without threats.

- Communications Security, a strategy that aims to secure information and information technology media.

- Network Security, a strategy that focuses on securing network equipment on organizational data.

Data security is a technique used to protect information from threats that might occur to guarantee information exchange [7]. It aims to reduce the level of risk and accelerate or maximize decision making. The level of security in information also depends on the level of sensitivity of the information in the database, information that is not too sensitive to the security system is not too tight, while for sensitive information, it is necessary to have a strict security level setting for access to that information [2], [8]-[11].

Cryptography is a technique used to protect data. Many techniques can be used to perform cryptographic processes. Cryptography involves the process of encryption and decryption [12]. The Data Encryption Standard (DES) algorithm is one technique that can be done to secure data [13]. This algorithm has a 64-bit encryption block. The key used is also 64-bit. The block length depends on the number of the plaintext used. This algorithm has a somewhat complicated process and stages. There is no complicated mathematical calculation process, but this algorithm has a substitution process that is complicated in several tables. By applying DES encryption, it is expected that the information sent will be safer and more secure [14]-[17].

\section{THEORIES:}

\subsection{Substitution Cipher}

Substitution ciphers are ciphers by substituting letters with other letters as specified. The main principle of substitution ciphers is to exchange each letter for plaintext with something [8], [18]-[20]. Substitution ciphers include classical cryptographic algorithms. The idea is to replace one or more letters in plaintext with one or more letters in plaintext with specific rules. These rules depend on the process of encryption and decryption. Substitution ciphers have several variants or types [21]. Types of substitution ciphers are: 
- Monoalphabetic Cipher. This type of substitution cipher is often also called a simple substitution cipher. The idea of single-alphabet substitution ciphers is to replace one character in plaintext into one character in the ciphertext with specific rules. The ciphering function is one to one function.

- Homophonic Substitution Cipher Cipher. The idea of a homophonic substitution cipher is to replace one character in plaintext into one or more characters in the ciphertext. The ciphering function is one to many functions.

- Alphalphabetic Substitution Cipher. This type of substitution cipher can be called a double substitution cipher. Multiple-alphabet substitution ciphers are single-alphabet substitution ciphers that use different keys. Therefore, compound-substitution ciphers have periods $\mathrm{m}, \mathrm{m}$ is the key length.

- Polygram Substitution Cipher. The idea of a polygram substitution cipher is to replace a character block with a ciphertext block. Blocks consist of one or more characters. For example, AAA was changed to BCD or PAP, and others.

\subsection{Data Encryption Standard (DES)}

Data Encryption Standard (DES) is a block cipher algorithm that is popular because it is used as a standard keysymmetry encryption algorithm, although at this time the standard has been replaced by a new algorithm, AES because DES is considered unsafe again. DES is the name of the symmetry encryption standard, the name of the encryption algorithm itself is DEA (Data Encryption Algorithm), but the DES name is more popular than DEA. The DES algorithm was developed at IBM under the leadership of W.L. Tuchman in 1972. This algorithm is based on the Lucifer algorithm created by Horst Feistel. This algorithm has been approved by the National Bureau of Standard (NBS) after its strength assessment by the United States National Security Agency (NSA) [22]-[24]

\section{METHODOLOGY:}

The DES algorithm requires several substitution tables. Tables are substitution, compression, expansion, inversion, and breakdown. These tables are used when performing encryption stages. The following are the tables used to perform DES encryption [25].

Table 1. Initial Permutation

\begin{tabular}{|l|l|l|l|l|l|l|l|}
\hline 58 & 50 & 42 & 34 & 26 & 18 & 10 & 2 \\
\hline 60 & 52 & 44 & 36 & 28 & 20 & 12 & 4 \\
\hline 62 & 54 & 46 & 38 & 30 & 22 & 14 & 6 \\
\hline 64 & 56 & 48 & 40 & 32 & 24 & 16 & 8 \\
\hline 57 & 49 & 41 & 33 & 25 & 17 & 9 & 1 \\
\hline 59 & 51 & 43 & 35 & 27 & 19 & 11 & 3 \\
\hline 61 & 53 & 45 & 37 & 29 & 21 & 13 & 5 \\
\hline 63 & 55 & 47 & 39 & 31 & 23 & 15 & 7 \\
\hline
\end{tabular}

Table 2. Permutation Compression 1

\begin{tabular}{|c|c|c|c|c|c|c|}
\hline 57 & 49 & 41 & 33 & 25 & 17 & 9 \\
\hline 1 & 58 & 50 & 42 & 34 & 26 & 18 \\
\hline 10 & 2 & 59 & 51 & 43 & 35 & 27 \\
\hline 19 & 11 & 3 & 60 & 52 & 44 & 36 \\
\hline 63 & 55 & 47 & 39 & 31 & 23 & 15 \\
\hline 7 & 62 & 54 & 45 & 38 & 30 & 22 \\
\hline 14 & 6 & 61 & 53 & 45 & 37 & 29 \\
\hline 21 & 13 & 5 & 28 & 20 & 12 & 4 \\
\hline
\end{tabular}

Table 3. Left Shift

\begin{tabular}{|c|c|}
\hline Round & $\begin{array}{c}\text { Number of Shifts (Left } \\
\text { Shift) }\end{array}$ \\
\hline 1 & 1 \\
\hline 2 & 1 \\
\hline 3 & 2 \\
\hline
\end{tabular}




\begin{tabular}{|c|c|}
\hline 4 & 2 \\
\hline 5 & 2 \\
\hline 6 & 2 \\
\hline 7 & 2 \\
\hline 8 & 2 \\
\hline 9 & 1 \\
\hline 10 & 2 \\
\hline 11 & 2 \\
\hline 12 & 2 \\
\hline 13 & 2 \\
\hline 14 & 2 \\
\hline 15 & 2 \\
\hline 16 & 1 \\
\hline
\end{tabular}

Table 4. Permutation Compression 2

\begin{tabular}{|c|c|c|c|c|c|}
\hline 14 & 17 & 11 & 24 & 1 & 5 \\
\hline 3 & 28 & 15 & 6 & 21 & 10 \\
\hline 23 & 19 & 12 & 4 & 26 & 8 \\
\hline 16 & 7 & 27 & 20 & 13 & 2 \\
\hline 41 & 52 & 31 & 37 & 47 & 55 \\
\hline 30 & 40 & 51 & 45 & 33 & 48 \\
\hline 44 & 49 & 39 & 56 & 34 & 53 \\
\hline 46 & 42 & 50 & 36 & 29 & 32 \\
\hline
\end{tabular}

Table 5. Permutation Compression 2

\begin{tabular}{|c|c|c|c|c|c|}
\hline 32 & 1 & 2 & 3 & 4 & 5 \\
\hline 4 & 5 & 6 & 7 & 8 & 9 \\
\hline 8 & 9 & 10 & 11 & 12 & 13 \\
\hline 12 & 13 & 14 & 15 & 16 & 17 \\
\hline 16 & 17 & 18 & 19 & 20 & 21 \\
\hline 20 & 21 & 22 & 23 & 24 & 25 \\
\hline 24 & 25 & 26 & 27 & 28 & 29 \\
\hline 28 & 29 & 30 & 31 & 32 & 1 \\
\hline
\end{tabular}

Table 6. S-Box

\begin{tabular}{|c|c|c|c|c|c|c|c|c|c|c|c|c|c|c|c|c|}
\hline \multicolumn{17}{|c|}{ S1 } \\
\hline & 0000 & 0001 & 0010 & 0011 & 0100 & 0101 & 0110 & 0111 & 1000 & 1001 & 1010 & 1011 & 1100 & 1101 & 1110 & 1111 \\
\hline 00 & 14 & 4 & 13 & 1 & 2 & 15 & 11 & 8 & 3 & 10 & 6 & 12 & 5 & 9 & 0 & 7 \\
\hline 01 & 0 & 15 & 7 & 4 & 14 & 2 & 13 & 1 & 10 & 7 & 12 & 11 & 9 & 5 & 3 & 8 \\
\hline 10 & 4 & 1 & 14 & 8 & 13 & 6 & 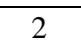 & 11 & 15 & 12 & 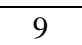 & 7 & 3 & 10 & 5 & 0 \\
\hline 11 & 15 & 12 & 8 & 2 & 4 & 9 & 1 & 7 & 5 & 11 & 3 & 14 & 10 & 0 & 6 & 13 \\
\hline \multicolumn{17}{|c|}{ S2 } \\
\hline & 0000 & 0001 & 0010 & 0011 & 0100 & 0101 & 0110 & 0111 & 1000 & 1001 & 1010 & 1011 & 1100 & 1101 & 1110 & 1111 \\
\hline 00 & 15 & 1 & 8 & 14 & 6 & 11 & 3 & 4 & 9 & 7 & 2 & 13 & 12 & 0 & 5 & 10 \\
\hline 01 & 3 & 13 & 4 & 7 & 15 & 2 & 8 & 14 & 12 & - & 1 & 10 & 6 & 9 & 11 & 5 \\
\hline 10 & 0 & 14 & 7 & 11 & 10 & 4 & 13 & 1 & 5 & 8 & 12 & 6 & 9 & 3 & 2 & 15 \\
\hline 11 & 13 & 8 & 10 & 1 & 3 & 15 & 4 & 2 & 11 & 6 & 7 & 12 & 0 & 5 & 14 & 9 \\
\hline \multicolumn{17}{|c|}{ S3 } \\
\hline & 0000 & 0001 & 0010 & 0011 & 0100 & 0101 & 0110 & 0111 & 1000 & 1001 & 1010 & 1011 & 1100 & 1101 & 1110 & 1111 \\
\hline 00 & 10 & 0 & 9 & 14 & 6 & 3 & 15 & 5 & 1 & 13 & 12 & 7 & 11 & 4 & 2 & 8 \\
\hline 01 & 13 & 7 & 0 & 9 & 3 & 4 & 6 & 10 & 2 & 8 & 5 & 14 & 12 & 11 & 15 & 1 \\
\hline 10 & 13 & 6 & 4 & 9 & 8 & 15 & 3 & 0 & 11 & 1 & 2 & 12 & 5 & 10 & 14 & 7 \\
\hline 11 & 1 & 10 & 13 & 0 & 6 & 9 & 8 & 7 & 4 & 15 & 14 & 3 & 11 & 5 & 2 & 12 \\
\hline \multicolumn{17}{|c|}{ S4 } \\
\hline & 0000 & 0001 & 0010 & 0011 & 0100 & 0101 & 0110 & 0111 & 1000 & 1001 & 1010 & 1011 & 1100 & 1101 & 1110 & 1111 \\
\hline
\end{tabular}


International Journal for InNovative Research in Multidisciplinary FieLd ISSN: 2455-0620 Volume - 4, Issue - 10, Oct - 2018 Monthly, Peer-Reviewed, Refereed, Indexed Journal with IC Value: 86.87

\begin{tabular}{|c|c|c|c|c|c|c|c|c|c|c|c|c|c|c|c|c|}
\hline 00 & 7 & 13 & 14 & 3 & 0 & 6 & 9 & 10 & 1 & 2 & 8 & 5 & 11 & 12 & 4 & 15 \\
\hline 01 & 13 & 8 & 11 & 5 & 6 & 15 & 0 & 3 & 4 & 7 & 2 & 12 & 1 & 10 & 14 & 9 \\
\hline 10 & 10 & 6 & 9 & 0 & 12 & 11 & 7 & 13 & 15 & 1 & 3 & 14 & 5 & 2 & 8 & 4 \\
\hline 11 & 3 & 15 & 0 & 6 & 10 & 1 & 13 & 18 & 9 & 4 & 5 & 11 & 12 & 7 & 2 & 14 \\
\hline \multicolumn{17}{|c|}{ S5 } \\
\hline & 0000 & 0001 & 0010 & 0011 & 0100 & 0101 & 0110 & 0111 & 1000 & 1001 & 1010 & 1011 & 1100 & 1101 & 1110 & 1111 \\
\hline 00 & 2 & 12 & 4 & 1 & 7 & 10 & 11 & 6 & 8 & 5 & 3 & 15 & 13 & 0 & 14 & 9 \\
\hline 01 & 14 & 11 & 2 & 12 & 4 & 7 & 13 & 1 & 5 & 0 & 15 & 10 & 3 & 9 & 8 & 15 \\
\hline 10 & 4 & 2 & 1 & 11 & 10 & 13 & 7 & 8 & 15 & 9 & 12 & 5 & 6 & 3 & 0 & 14 \\
\hline 11 & 11 & 8 & 12 & 7 & 1 & 14 & 2 & 13 & 6 & 15 & 0 & 9 & 10 & 4 & 5 & 3 \\
\hline \multicolumn{17}{|c|}{ S6 } \\
\hline & 0000 & 0001 & 0010 & 0011 & 0100 & 0101 & 0110 & 0111 & 1000 & 1001 & 1010 & 1011 & 1100 & 1101 & 1110 & 1111 \\
\hline 00 & 12 & 1 & 10 & 15 & 9 & 2 & 6 & 8 & 0 & 13 & 3 & 4 & 14 & 7 & 5 & 11 \\
\hline 01 & 10 & 15 & 4 & 2 & 7 & 12 & 9 & 5 & 6 & 1 & 13 & 14 & 0 & 11 & 3 & 8 \\
\hline 10 & 9 & 14 & 15 & 5 & 2 & 8 & 12 & 3 & 7 & 0 & 4 & 10 & 1 & 13 & 11 & 6 \\
\hline 11 & 4 & 3 & 2 & 12 & 9 & 5 & 15 & 10 & 11 & 14 & 1 & 7 & 6 & 0 & 8 & 13 \\
\hline \multicolumn{17}{|c|}{ S7 } \\
\hline & 0000 & 0001 & 0010 & 0011 & 0100 & 0101 & 0110 & 0111 & 1000 & 1001 & 1010 & 1011 & 1100 & 1101 & 1110 & 1111 \\
\hline 00 & 4 & 11 & 2 & 14 & 15 & 0 & 8 & 13 & 3 & 12 & 9 & 7 & 5 & 10 & 6 & 1 \\
\hline 01 & 13 & 0 & 11 & 7 & 4 & 9 & 1 & 10 & 14 & 3 & 5 & 12 & 2 & 15 & 8 & 6 \\
\hline 10 & 1 & 4 & 11 & 13 & 12 & 3 & 7 & 14 & 10 & 15 & 6 & 8 & 0 & 5 & 9 & 2 \\
\hline 11 & 6 & 11 & 13 & 8 & 1 & 4 & 10 & 7 & 9 & 5 & 0 & 15 & 14 & 2 & 3 & 12 \\
\hline \multicolumn{17}{|c|}{ S8 } \\
\hline & 0000 & 0001 & 0010 & 0011 & 0100 & 0101 & 0110 & 0111 & 1000 & 1001 & 1010 & 1011 & 1100 & 1101 & 1110 & 1111 \\
\hline 00 & 13 & 2 & 8 & 4 & 6 & 15 & 11 & 1 & 10 & 9 & 3 & 14 & 5 & 0 & 12 & 7 \\
\hline 01 & 1 & 15 & 13 & 8 & 10 & 3 & 7 & 4 & 12 & 5 & 6 & 11 & 0 & 14 & 9 & 2 \\
\hline 10 & 7 & 11 & 4 & 1 & 9 & 12 & 14 & 2 & 0 & 6 & 10 & 13 & 15 & 3 & 5 & 8 \\
\hline 11 & 2 & 1 & 14 & 7 & 4 & 10 & 8 & 13 & 15 & 12 & 9 & 0 & 3 & 5 & 6 & 11 \\
\hline
\end{tabular}

Table 7. Permutation

\begin{tabular}{|c|c|c|c|c|c|c|c|}
\hline 16 & 7 & 20 & 21 & 29 & 12 & 28 & 17 \\
\hline 1 & 15 & 23 & 26 & 5 & 18 & 31 & 10 \\
\hline 2 & 8 & 24 & 14 & 32 & 27 & 3 & 9 \\
\hline 19 & 13 & 30 & 6 & 22 & 11 & 4 & 25 \\
\hline
\end{tabular}

Table 8. Inverse Permutation

\begin{tabular}{|l|l|l|l|l|l|l|l|}
\hline 40 & 8 & 48 & 16 & 56 & 24 & 64 & 32 \\
\hline 39 & 7 & 47 & 15 & 55 & 23 & 63 & 31 \\
\hline 38 & 6 & 46 & 14 & 54 & 22 & 62 & 30 \\
\hline 37 & 5 & 45 & 13 & 53 & 21 & 61 & 29 \\
\hline 36 & 4 & 44 & 12 & 52 & 20 & 60 & 28 \\
\hline 35 & 3 & 43 & 11 & 51 & 19 & 59 & 27 \\
\hline 34 & 2 & 42 & 10 & 50 & 18 & 58 & 26 \\
\hline 33 & 1 & 41 & 9 & 49 & 17 & 57 & 25 \\
\hline
\end{tabular}

\section{RESULT AND DISCUSSION}

This section discusses testing the DES algorithm. The test is done by giving plaintext and keys each of eight characters. The following is a step illustration of the DES algorithm.

Step 1

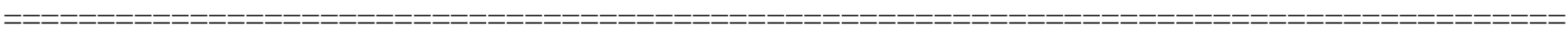
$===$

Plaintext =

\begin{tabular}{|c|c|c|c|c|c|c|c|c|}
\cline { 2 - 9 } \multicolumn{1}{c|}{} & C & O & M & P & U & T & E & R \\
\hline DEC & 67 & 79 & 77 & 80 & 85 & 84 & 69 & 82 \\
\hline HEX & 43 & $4 \mathrm{~F}$ & $4 \mathrm{D}$ & 50 & 55 & 54 & 45 & 52 \\
\hline
\end{tabular}


Key

$=$

\begin{tabular}{|l|l|l|l|l|l|l|l|l|}
\hline HEX & 13 & 34 & 57 & 79 & $9 \mathrm{~B}$ & $\mathrm{BC}$ & $\mathrm{DF}$ & $\mathrm{F} 1$ \\
\hline
\end{tabular}

Step 2

\begin{tabular}{|c|c|}
\hline & \\
\hline Plain Bit & $\begin{array}{l}=0100001101001111010011010101000001010101010101000100010101010010 \\
=\text { LR is the PLAIN BIT value based on the order in the Permutation Table }\end{array}$ \\
\hline LR & $\begin{array}{l}=1111111110111000011101100101011100000000000000000000011010000011 \\
=\text { Separate LR into } 2 \text { parts L and R }\end{array}$ \\
\hline [0] & $=11111111101110000111011001010111$ \\
\hline [0] & $=00000000000000000000011010000011$ \\
\hline
\end{tabular}

Step 3: Generate Key

\begin{tabular}{|c|c|}
\hline \multicolumn{2}{|c|}{$===$} \\
\hline Key & 0110011010001010111011110011001101110111100110111111111000 \\
\hline & $=\mathrm{CD}$ is the KEY BIT value based on the order in the PC1 Table \\
\hline $\mathrm{CD}$ & $\begin{array}{l}=11110000110011001010101011110101010101100110011110001111 \\
=\text { Separate CD into } 2 \text { parts C and D }\end{array}$ \\
\hline $\mathrm{C}[0]$ & $=1111000011001100101010101111$ \\
\hline$[0]$ & $=0101010101100110011110001111$ \\
\hline
\end{tabular}

Step 4: Slide bits C and D according to the sliding distance in the LeftShift Table

$\begin{array}{llll}======================================================= \\ === \\ \mathrm{C}[0] & =1111000011001100101010101111 & \mathrm{D}[0] & =0101010101100110011110001111 \\ \mathrm{C}[1] & =1110000110011001010101011111 & \mathrm{D}[1] & =1010101011001100111100011110 \\ \mathrm{C}[2] & =1100001100110010101010111111 & \mathrm{D}[2] & =0101010110011001111000111101 \\ \mathrm{C}[3] & =0000110011001010101011111111 & \mathrm{D}[3] & =0101011001100111100011110101 \\ \mathrm{C}[4] & =0011001100101010101111111100 & \mathrm{D}[4] & =0101100110011110001111010101 \\ \mathrm{C}[5] & =1100110010101010111111110000 & \mathrm{D}[5] & =0110011001111000111101010101 \\ \mathrm{C}[6] & =0011001010101011111111000011 & \mathrm{D}[6] & =1001100111100011110101010101 \\ \mathrm{C}[7] & =1100101010101111111100001100 & \mathrm{D}[7] & =0110011110001111010101010110 \\ \mathrm{C}[8] & =001010101011111110000110011 & \mathrm{D}[8] & =1001111000111101010101011001 \\ \mathrm{C}[9] & =0101010101111111100001100110 & \mathrm{D}[9] & =001111000111010101010110011 \\ \mathrm{C}[10] & =0101010111111110000110011001 & \mathrm{D}[10] & =1111000111101010101011001100 \\ \mathrm{C}[11] & =0101011111111000011001100101 & \mathrm{D}[11] & =1100011110101010101100110011 \\ \mathrm{C}[12] & =010111111100001100110010101 & \mathrm{D}[12] & =0001111010101010110011001111 \\ \mathrm{C}[13] & =0111111110000110011001010101 & \mathrm{D}[13] & =0111101010101011001100111100 \\ \mathrm{C}[14] & =1111111000011001100101010101 & \mathrm{D}[14] & =1110101010101100110011110001 \\ \mathrm{C}[15] & =1111100001100110010101010111 & \mathrm{D}[15] & =1010101010110011001111000111 \\ \mathrm{C}[16] & =1111000011001100101010101111 & \mathrm{D}[16] & =0101010101100110011110001111\end{array}$

Step 5: $\mathrm{K}$ is a $\mathrm{CD}$ value based on the order in the PC2 table (64 to 48 bits)

\begin{tabular}{|c|c|}
\hline \\
\hline \multicolumn{2}{|r|}{$=11001011001111011000101100001110000101111111010$} \\
\hline $\mathrm{K}[1]$ & $=000110110000001011101111111111000111000001110010$ \\
\hline $\mathrm{K}[2]$ & $=011110011010111011011001110110111100100111100101$ \\
\hline $\mathrm{K}[3]$ & $=010101011111110010001010010000101100111110011001$ \\
\hline $\mathrm{K}[4]$ & $=011100101010110111010110110110110011010100011101$ \\
\hline $\mathrm{K}[5]$ & $=011111001110110000000111111010110101001110101000$ \\
\hline $\mathrm{K}[6]$ & $=011000111010010100111110010100000111101100101111$ \\
\hline $\mathrm{K}[7]$ & $=111011001000010010110111111101100001100010111100$ \\
\hline $\mathrm{K}[8]$ & $=111101111000101000111010110000010011101111111011$ \\
\hline
\end{tabular}


$\mathrm{K}[9]=111000001101101111101011111011011110011110000001$

$\mathrm{K}[10]=101100011111001101000111101110100100011001001111$

$\mathrm{K}[11]=001000010101111111010011110111101101001110000110$

$\mathrm{K}[12]=011101010111000111110101100101000110011111101001$

$\mathrm{K}[13]=100101111100010111010001111110101011101001000001$

$\mathrm{K}[14]=010111110100001110110111111100101110011100111010$

$\mathrm{K}[15]=101111111001000110001101001111010011111100001010$

$\mathrm{K}[16]=110010110011110110001011000011100001011111110101$

Step 6: Expansion

$==$

$=\mathrm{ER}$ is the $\mathrm{R}$ value based on the order in the Expansion Table (32 to 48 bits)

$=\mathrm{B}$ is a meeting between rows (bits 1,6) and columns (bits 2 to 5) in the S-BOX Table

$=\mathrm{B}$ produces 32 bits

The S-Box is a vital part of DES because it is the most challenging part to solve. It is because the S-Box is the only part of DES whose computation is not linear. Meanwhile, the design of the S-Box itself was not made public. Because of this, many suspect that the S-Box was designed in such a way as to provide a trapdoor.

Iteration 1

\begin{tabular}{|c|c|}
\hline & $=\mathrm{L}$ is \\
\hline$[0]$ & $\begin{array}{l}=11111111101110000111011001010111 \\
=\mathrm{R} \text { is } \mathrm{PB} \text { xor previous } \mathrm{L}\end{array}$ \\
\hline $\mathrm{R}[0]$ & $=00000000000000000000011010000011$ \\
\hline ER[0] & $=100000000000000000000000000000001101010000000110$ \\
\hline $\mathrm{K}[1]$ & $\begin{array}{l}=000110110000001011101111111111000111000001110010 \\
=\mathrm{LA} \text { is an XOR function on ER against K } \\
=\end{array}$ \\
\hline [1] & 1001010010001110100 \\
\hline $3[1]$ & $\begin{array}{l}101010 \\
\text { the Permutation table }\end{array}$ \\
\hline & $=00101000101100110100010011010001$ \\
\hline
\end{tabular}

Iteration 2

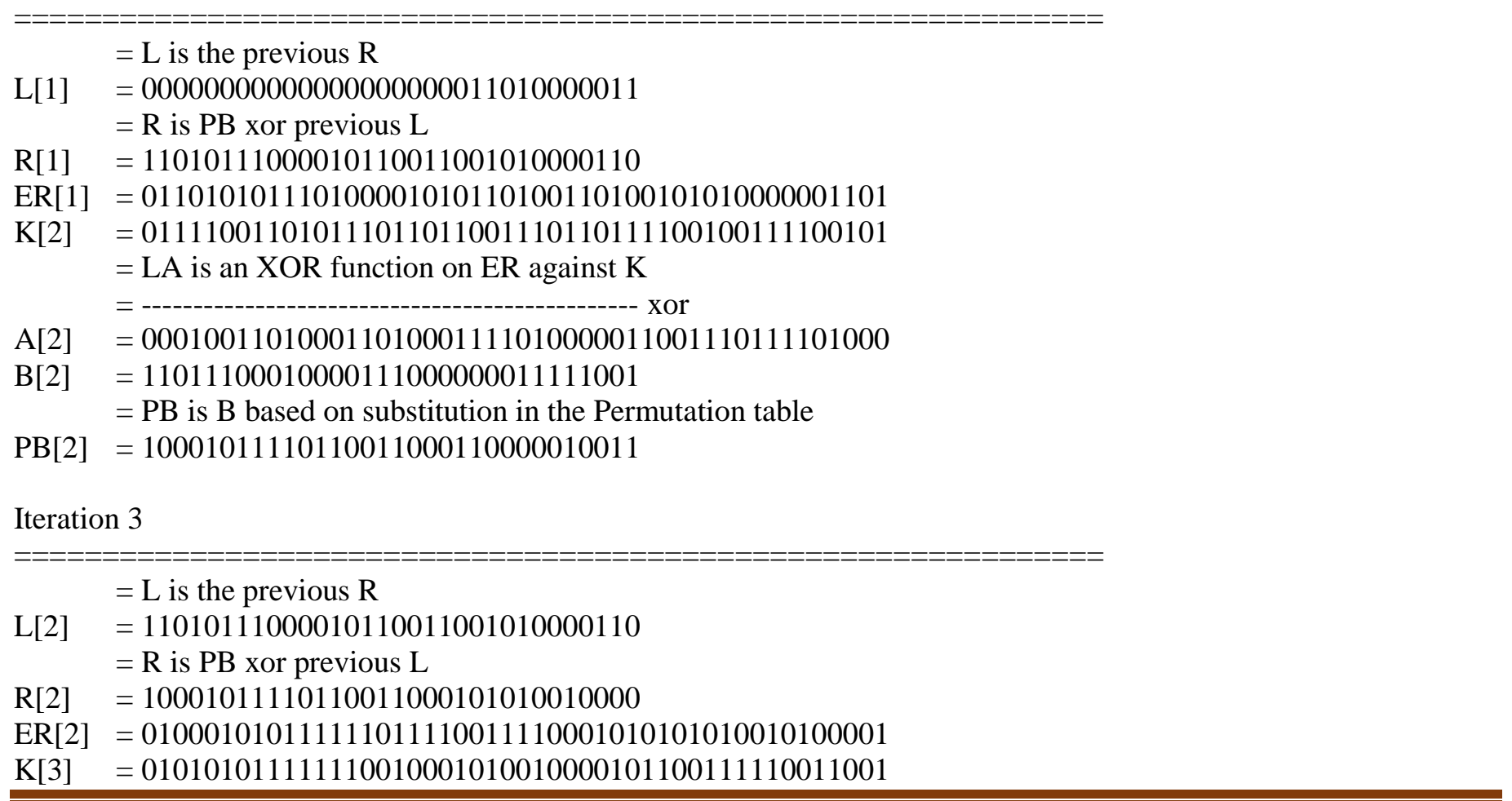


$=\mathrm{LA}$ is an XOR function on ER against $\mathrm{K}$

= ----------------------------------------------- xor

$\mathrm{A}[3]=000100001000001001111001100001111001101100111000$

$\mathrm{B}[3]=11010110001111001011011001111111$

$=\mathrm{PB}$ is $\mathrm{B}$ based on substitution in the Permutation table

$\mathrm{PB}[3]=01101111101100101001110011111110$

Iteration 4

\begin{tabular}{|c|c|}
\hline & $=\mathrm{L}$ is the previous $\mathrm{R}$ \\
\hline L[3] & $\begin{array}{l}=10001011110110011000101010010000 \\
=\mathrm{R} \text { is } \mathrm{PB} \text { xor previous } \mathrm{L}\end{array}$ \\
\hline $\mathrm{R}[3]$ & $=10111000101110011010111001111000$ \\
\hline ER[3] & $=010111110001010111110011110101011100001111110001$ \\
\hline $\mathrm{K}[4]$ & $\begin{array}{l}=011100101010110111010110110110110011010100011101 \\
=\mathrm{LA} \text { is an XOR function on ER against K } \\
=\text {-------- xor }\end{array}$ \\
\hline $\mathrm{A}[4]$ & $=001011011011100000100101000011101111011011101100$ \\
\hline $\mathrm{B}[4]$ & $\begin{array}{l}=00101001110100001011101011111110 \\
=\mathrm{PB} \text { is } \mathrm{B} \text { based on substitution in the Permutation table }\end{array}$ \\
\hline $\mathrm{B}[4]$ & $=00111111001110110100011110100001$ \\
\hline
\end{tabular}

Iteration 5

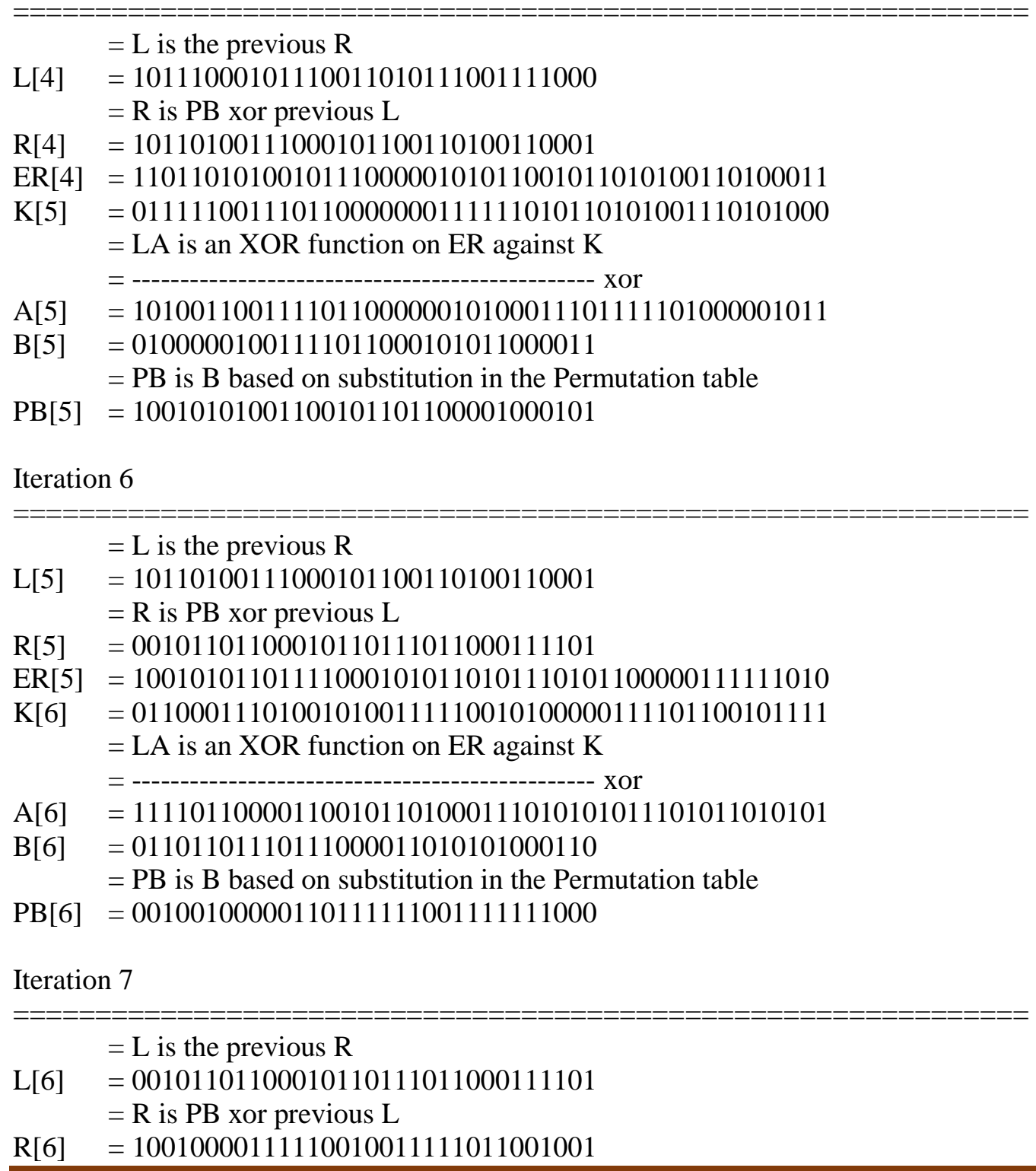


$\mathrm{ER}[6]=110010100001011111110010100111111101011001010011$

$\mathrm{K}[7]=111011001000010010110111111101100001100010111100$

$=\mathrm{LA}$ is an XOR function on ER against $\mathrm{K}$

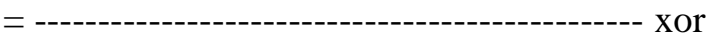

$\mathrm{A}[7]=001001101001001101000101011010011100111011101111$

$\mathrm{B}[7]=11100011011010110000010100101101$

$=\mathrm{PB}$ is $\mathrm{B}$ based on substitution in the Permutation table

$\mathrm{PB}[7]=11001000110000011110111001101100$

Iteration 8

\begin{tabular}{|c|c|}
\hline L[7] & $\begin{array}{l}=\mathrm{L} \text { is the previous } \mathrm{R} \\
=10010000111110010011111011001001 \\
=\mathrm{R} \text { is } \mathrm{PB} \text { xor previous } \mathrm{L}\end{array}$ \\
\hline $\mathrm{R}[7]$ & 1001100001010001 \\
\hline ER[7] & 1010100111100000 \\
\hline $\mathrm{K}[8]$ & $\begin{array}{l}=111101111000101000111010110000010011101111111011 \\
=\text { LA is an XOR function on ER against K } \\
=\end{array}$ \\
\hline $\mathrm{A}[8]$ & 001000000110111110001110001110010101100 \\
\hline $\mathrm{B}[8]$ & $\begin{array}{l}=00001000110110001000001111010101 \\
=\mathrm{PB} \text { is } \mathrm{B} \text { based on substitution in the Permutation table }\end{array}$ \\
\hline [8] & 1110010010100101100001 \\
\hline
\end{tabular}

Iteration 9

\begin{tabular}{|c|c|}
\hline & $=\mathrm{L}$ is the previous $\mathrm{R}$ \\
\hline $\mathrm{L}[8]$ & $\begin{array}{l}=11100101010010101001100001010001 \\
=\mathrm{R} \text { is } \mathrm{PB} \text { xor previous } \mathrm{L}\end{array}$ \\
\hline $\mathrm{R}[8]$ & $=10010111110000000001011110101000$ \\
\hline ER[8] & $=010010101111111000000000000010101111110101010001$ \\
\hline $\mathrm{K}[9]$ & $\begin{array}{l}=111000001101101111101011111011011110011110000001 \\
=\text { LA is an XOR function on ER against K } \\
=\end{array}$ \\
\hline $\mathrm{A}[9]$ & $=101010100010010111101011111001110001101011010000$ \\
\hline $\mathrm{B}[9]$ & $\begin{array}{l}=01101110111000011010101101001010 \\
=\mathrm{PB} \text { is } \mathrm{B} \text { based on substitution in the Permutation table }\end{array}$ \\
\hline $\mathrm{PB}[9]$ & $=11011001001110111010001110010100$ \\
\hline \multicolumn{2}{|c|}{$\begin{array}{l}\text { Iteration } 10 \\
========\end{array}$} \\
\hline $\mathrm{L}[9]$ & $\begin{array}{l}=\mathrm{L} \text { is the previous } \mathrm{R} \\
=10010111110000000001011110101000 \\
=\mathrm{R} \text { is } \mathrm{PB} \text { xor previous } \mathrm{L}\end{array}$ \\
\hline $\mathrm{R}[9]$ & $=00111100011100010011101111000101$ \\
\hline $\mathrm{ER}[9]$ & $=100111111000001110100010100111110111111000001010$ \\
\hline $\mathrm{K}[10]$ & $\begin{array}{l}=101100011111001101000111101110100100011001001111 \\
=\text { LA is an XOR function on ER against K } \\
=\end{array}$ \\
\hline $\mathrm{A}[10]$ & $=001011100111000011100101001001010011100001000101$ \\
\hline $\mathrm{B}[10]$ & $\begin{array}{l}=00100001011100000100000101101101 \\
=\mathrm{PB} \text { is } \mathrm{B} \text { based on substitution in the Permutation table }\end{array}$ \\
\hline $\mathrm{PB}[10]$ & $=00001100000101010110111000100100$ \\
\hline \multicolumn{2}{|c|}{ Iteration 11} \\
\hline L[10] & $\begin{array}{l}=\mathrm{L} \text { is the previous } \mathrm{R} \\
=00111100011100010011101111000101\end{array}$ \\
\hline
\end{tabular}


$=\mathrm{R}$ is $\mathrm{PB}$ xor previous $\mathrm{L}$

$\mathrm{R}[10]=10011011110101010111100110001100$

$\mathrm{ER}[10]=010011110111111010101010101111110011110001011001$

$\mathrm{K}[11]=001000010101111111010011110111101101001110000110$

$=\mathrm{LA}$ is an XOR function on ER against $\mathrm{K}$

= ------------------------------------------------ xor

$\mathrm{A}[11]=011011100010000101111001011000011110111111011111$

$\mathrm{B}[11]=01011110000011001101101111000010$

$=\mathrm{PB}$ is $\mathrm{B}$ based on substitution in the Permutation table

$\mathrm{PB}[11]=01110001001111101011000001010011$

Iteration 12

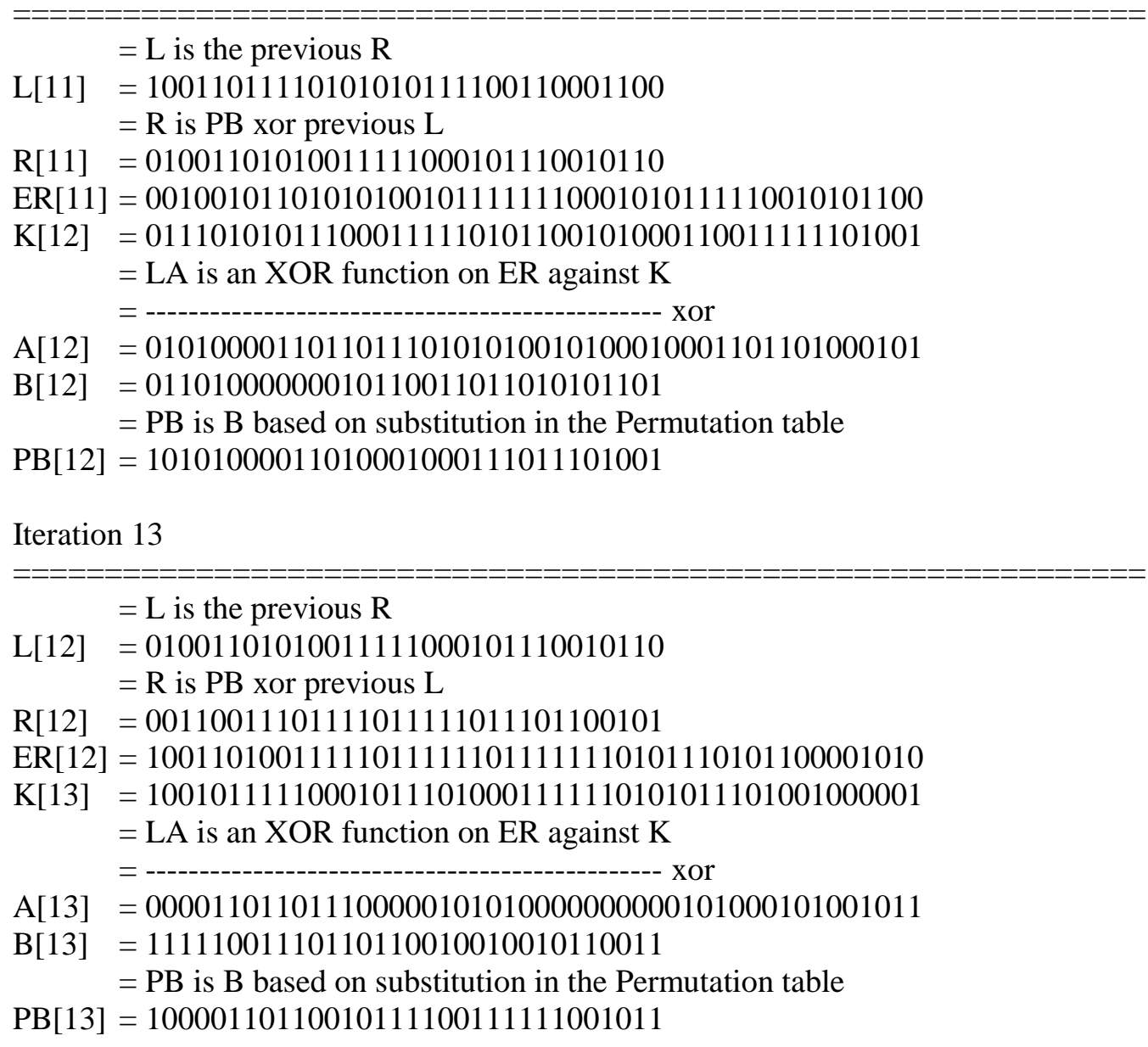

Iteration 14

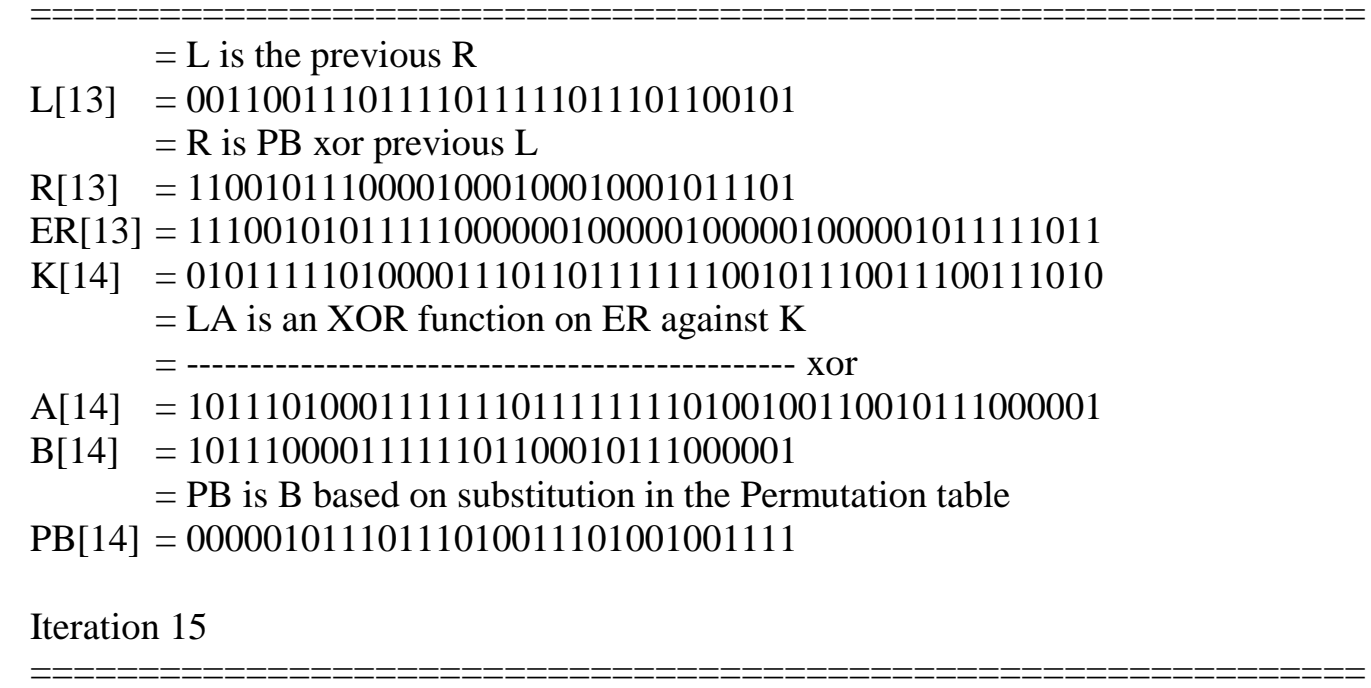


$=\mathrm{L}$ is the previous $\mathrm{R}$

$\mathrm{L}[14]=11001011100001000100010001011101$

$=\mathrm{R}$ is $\mathrm{PB}$ xor previous $\mathrm{L}$

$\mathrm{R}[14]=00110110011000001100110100101010$

$\mathrm{ER}[14]=000110101100001100000001011001011010100101010100$

$\mathrm{K}[15]=101111111001000110001101001111010011111100001010$

$=\mathrm{LA}$ is an XOR function on ER against $\mathrm{K}$

= -

$\mathrm{A}[15]=101001010101001010001100010110001001011001011110$

$\mathrm{B}[15]=01000001001110011111011100100111$

$=\mathrm{PB}$ is $\mathrm{B}$ based on substitution in the Permutation table

$\mathrm{PB}[15]=10100101001001101110110011101100$

Iteration 16

\begin{tabular}{|c|c|}
\hline & $=\mathrm{L}$ is the previous $\mathrm{R}$ \\
\hline $\mathrm{L}[15]$ & $\begin{array}{l}=00110110011000001100110100101010 \\
=\mathrm{R} \text { is } \mathrm{PB} \text { xor previous } \mathrm{L}\end{array}$ \\
\hline $\mathrm{R}[15]$ & $=01101110101000101010100010110001$ \\
\hline ER[15] & $=101101011101010100000101010101010001010110100010$ \\
\hline $\mathrm{K}[16]$ & $\begin{array}{l}=110010110011110110001011000011100001011111110101 \\
=\text { LA is an XOR function on ER against K } \\
=\end{array}$ \\
\hline $\mathrm{A}[16]$ & $=011111101110100010001110010110110000001001010111$ \\
\hline $\mathrm{B}[16]$ & $\begin{array}{l}=10000001011010101111011101001011 \\
=\mathrm{PB} \text { is } \mathrm{B} \text { based on substitution in the Permutation table }\end{array}$ \\
\hline $\mathrm{PB}[16]$ & $=00101001111101110110100011001100$ \\
\hline & $=\mathrm{L}$ is the previous $\mathrm{R}$ \\
\hline $\mathrm{L}[16]$ & $\begin{array}{l}=01101110101000101010100010110001 \\
=\mathrm{R} \text { is } \mathrm{PB} \text { xor previous } \mathrm{L}\end{array}$ \\
\hline $\mathrm{R}[16]$ & $=00011111100101111010010111100110$ \\
\hline
\end{tabular}

Step 7: Join LR

\begin{tabular}{|c|c|}
\hline & \\
\hline RL & $\begin{array}{l}=0001111110010111101001011110011001101110101000101010100010110001 \\
=\mathrm{CT} \text { is an RL value based on substitution in the Inverse Permutation table }\end{array}$ \\
\hline CT & $=0101011011110001110101011100100001010010101011111000000100111111$ \\
\hline Hex & $=56 \mathrm{~F} 1 \mathrm{D} 5 \mathrm{C} 852 \mathrm{AF} 813 \mathrm{~F}$ \\
\hline
\end{tabular}

Ciphertext $=$

\begin{tabular}{|c|c|c|c|c|c|c|c|c|}
\cline { 2 - 9 } \multicolumn{1}{c|}{} & $\mathbf{1 3}$ & $\mathbf{3 4}$ & $\mathbf{5 7}$ & $\mathbf{7 9}$ & $\mathbf{9 B}$ & $\mathbf{B C}$ & DF & F1 \\
\hline DEC & 19 & 52 & 87 & 121 & 155 & 188 & 223 & 241 \\
\hline CHAR & $! !$ & 4 & $\mathrm{~W}$ & $\mathrm{y}$ & $>$ & $1 / 4$ & $\beta$ & $\tilde{\mathrm{n}}$ \\
\hline
\end{tabular}

\section{CONCLUSION:}

DES is a block cipher that operates with a 64-bit size. This algorithm is included in the substitution algorithm which is difficult to solve because it has 16 iterations during the encryption process. The DES algorithm can secure messages securely enough and give the Avalanche effect where each character that is secured using this DES algorithm is entirely unreadable and disappears on the ciphertext. The eight character key length used in the Data Encryption Standard algorithm can be said to be quite safe because when tested using the brute force method it takes more than 10 hours on average to find the password used. The use of this algorithm is beneficial for users in sending information on global networks. This algorithm does not require complicated mathematical calculations. This algorithm works by substituting the position of the plaintext matrix and the key to create a new character that is a ciphertext. 


\section{REFERENCES:}

1. A. H. Lubis, S. Z. S. Idrus, and A. Sarji, "ICT Usage Amongst Lecturers and Its Impact Towards Learning Process Quality," vol. 34, no. 1, pp. 284-299, 2018.

2. A. P. U. Siahaan, "Rabin-Karp Elaboration in Comparing Pattern Based on Hash Data," Int. J. Secur. Its Appl., vol. 12, no. 2, pp. 59-66, Mar. 2018.

3. M. D. T. P. Nasution, Y. Rossanty, A. P. U. Siahaan, and S. Aryza, "The Phenomenon of Cyber-Crime and Fraud Victimization in Online Shop," Int. J. Civ. Eng. Technol., vol. 9, no. 6, pp. 1583-1592, 2018.

4. H. Ming and S. LiZhong, "A New System Design of Network Invasion Forensics," in 2009 Second International Conference on Computer and Electrical Engineering, 2009, pp. 596-599.

5. B. Forouzan, Cryptography and Network Security. New York, NY, USA: McGraw-Hill, 2006.

6. A. Lubis and A. P. U. Siahaan, "Network Forensic Application in General Cases," IOSR J. Comput. Eng., vol. 18, no. 6, pp. 41-44, 2016.

7. W. Stallings, Cryptography and Network Security Principles and Practices, 4th ed. Prentice Hall, 2005.

8. M. Syahrizal, M. Murdani, S. D. Nasution, M. Mesran, R. Rahim, and A. P. U. Siahaan, "Modified Playfair Cipher Using Random Key Linear Congruent Method," J. Online Jar. COT POLIPD, vol. 10, no. 2, pp. 45-49, 2017.

9. I. Sumartono, A. P. U. Siahaan, and Arpan, "Base64 Character Encoding and Decoding Modeling," Int. J. Recent Trends Eng. Res., vol. 2, no. 12, pp. 63-68, 2016.

10. S. Ramadhani, Y. M. Saragih, R. Rahim, and A. P. U. Siahaan, "Post-Genesis Digital Forensics Investigation," Int. J. Sci. Res. Sci. Technol., vol. 3, no. 6, pp. 164-166, 2017.

11. Khairul et al., "Effect of Matrix Size in Affecting Noise Reduction Level of Filtering," Int. J. Eng. Technol., vol. 7, no. 3, pp. 1272-1275, 2018.

12. A. P. U. Siahaan, How to Code: Advanced Encryption Standard in C\#. Medan: Fakultas Ekonomi Universitas Panca Budi, 2018.

13. M. Sharma and R. B. Garg, "DES: The oldest symmetric block key encryption algorithm," in 2016 International Conference System Modeling \& Advancement in Research Trends (SMART), 2016, pp. 53-58.

14. A. P. U. Siahaan, "Three-Pass Protocol Concept in Hill Cipher Encryption Technique," Int. J. Sci. Res., vol. 5, no. 7, pp. 1149-1152, 2016.

15. A. P. U. Siahaan, "Factorization Hack of RSA Secret Numbers."

16. Hariyanto and A. P. U. Siahaan, "Intrusion Detection System in Network Forensic Analysis and," IOSR J. Comput. Eng., vol. 18, no. 6, pp. 115-121, 2016.

17. V. Tasril, M. B. Ginting, Mardiana, and A. P. U. Siahaan, "Threats of Computer System and its Prevention," Int. J. Sci. Res. Sci. Technol., vol. 3, no. 6, pp. 448-451, 2017.

18. B. Kapoor, P. Pandya, and J. S. Sherif, “Cryptography," Kybernetes, vol. 40, no. 9/10, pp. 1422-1439, Oct. 2011.

19. W. Fitriani, R. Rahim, B. Oktaviana, and A. P. U. Siahaan, "Vernam Encypted Text in End of File Hiding Steganography Technique,” Int. J. Recent Trends Eng. Res., vol. 3, no. 7, pp. 214-219, Jul. 2017.

20. I. Sumartono, A. P. U. Siahaan, and N. Mayasari, "An Overview of the RC4 Algorithm," IOSR J. Comput. Eng., vol. 18, no. 6, pp. 67-73, 2016.

21. B. Parmaza, "Substitution Cipher," 2018. [Online]. Available: http://itjambi.com/cipher-substitusi-substitutioncipher/. [Accessed: 10-Oct-2018].

22. Zae, "Algoritma DES (Data Encryption Standard)," 2009. [Online]. Available: http://ilmukriptografi.blogspot.com/2009/05/algoritma-des-data-encryption-standart.html.

23. Zae, "Tutorial Pemrograman Kriptografi C++ dan Java," Belajar Kriptografi Sambil Ngopi, 2015. [Online]. Available: http://ilmu-kriptografi.blogspot.com/2009/05/fungsi-hash.html.

24. A. Singh, M. Marwaha, B. Singh, and S. Singh, "Comparative Study of DES, 3DES, AES and RSA," Int. J. Comput. Technol., vol. 9, no. 3, pp. 1162-1170, Dec. 2010.

25. Zacky, "Enkripsi Algoritma DES (Data Encryption Standard)," KRIPTOGRAFI \& JARINGAN KOMPUTER, 2016. [Online]. Available: http://kriptografijaringan.blogspot.com/2016/03/enskripsi-algoritma-des-dataencryption.html. 